\title{
Transcriptome profiling of the subventricular zone and dentate gyrus in an animal model of Parkinson's disease
}

\author{
XIN-JIE BAO ${ }^{1 *}$, GENG-CHAO WANG ${ }^{2 *}$, FU-XING ZUO $^{3 *}$, XUE-YUAN LI ${ }^{1}$, JUN WU $^{4}$, GUO CHEN $^{4}$, \\ WAN-CHEN DOU ${ }^{1}$, YI GUO ${ }^{1}$, QIN SHEN ${ }^{4}$ and REN-ZHI WANG ${ }^{1}$
}

\author{
${ }^{1}$ Department of Neurosurgery, Peking Union Medical College Hospital, Chinese Academy of Medical Sciences \\ and Peking Union Medical College; ${ }^{2}$ State Key Laboratory of Medical Molecular Biology and Department of Biochemistry \\ and Molecular Biology, Institute of Basic Medical Sciences, Chinese Academy of Medical Sciences and Peking Union Medical \\ College, Beijing 100730; ${ }^{3}$ Department of Neurosurgery, National Cancer Center/Cancer Hospital, \\ Chinese Academy of Medical Sciences and Peking Union Medical College, Beijing 100021; \\ ${ }^{4}$ Center for Stem Cell Biology and Regenerative Medicine, Center for Life Sciences, \\ School of Medicine, Tsinghua University, Beijing 100084, P.R. China
}

Received March 29, 2016; Accepted June 20, 2017

DOI: $10.3892 /$ ijmm.2017.3052

\begin{abstract}
Adult neurogenesis in the subventricular zone (SVZ), as well as in the subgranular zone contributes to brain maintenance and regeneration. In the adult brain, dopamine (DA) can regulate the endogenous neural stem cells within these two regions, while a DA deficit may affect neurogenesis. Notably, the factors that regulate in vivo neurogenesis in these subregions have not yet been fully characterized, particularly following DA depletion. In thi study, we performed RNA sequencing to investigate transcriptomic changes in the SVZ and dentate gyrus (DG) of mice in response to 1-methyl4-phenyl-1,2,3,6-tetrahydropyridine (MPTP). This analysis identified differentially expressed genes which were involved in the regulation of transcription, immune response, extracellular region, cell junction and myelination. These genes partially displayed different temporal profiles of expression, some of which may participate in the metabolic switch related to neurogenesis. Additionally, the mitogen-activated protein kinase (MAPK) signaling pathway was shown to be been positively regulated in the SVZ, while it was negatively affected in the DG following MPTP administration. Overall, our findings indicate that exposure to MPTP may exert different effects on transcriptome profiling between the SVZ and DG.
\end{abstract}

Correspondence to: Dr Ren-Zhi Wang, Department of Neurosurgery, Peking Union Medical College Hospital, Chinese Academy of Medical Sciences and Peking Union Medical College, 1 Shuaifuyuan, Dongcheng, Beijing 100730, P.R. China

E-mail:wangrz@126.com

\section{*Contributed equally}

Key words: 1-methyl-4-phenyl-1,2,3,6-tetrahydropyridine, neurogenesis, subventricular zone, dentate gyrus, RNA sequencing

\section{Introduction}

Active neurogenesis occurs throughout life in the subventricular zone (SVZ) of the lateral ventricle, as well as in the subgranular zone (SGZ) of the dentate gyrus (DG) (1). Currently, scientific interest in endogenous repair has mostly focused on these two regions that harbor multipotent neural stem cells (NSCs), which can give rise to interneurons and contribute to neural plasticity $(1,2)$. However, it is unclear how distinct pools of NSCs may react to neurodegeneration. In addition, the molecular mechanisms underlying the process are not yet well understood.

Parkinson's disease (PD) is a common neurodegenerative disorder, characterized by the progressive loss of dopaminergic neurons and consequent dopamine (DA) deficit. In the adult brain, DA contributes to the regulation of endogenous NSCs, and DA depletion may affect neurogenesis (2-4). However, neurogenesis profiling that is activated or inhibited has been a subject of heated debate. Several findings have provided evidence that DA denervation reduces cell proliferation, differentiation and migration in the SVZ and SGZ in individuals with $\mathrm{PD}$, as well as in mice administered 1-methyl-4-phenyl-1,2,3,6tetrahydropyridine (MPTP) $(2,5)$, while others have emphasized that the proliferative capacity of the SVZ and SGZ is not diminished $(4,5)$ or even increased $(2,6,7)$ in the parkinsonian brain. Notably, there are several essential signaling pathways regulating proliferation, differentiation and migration, as well as the survival of neural precursor cells (NPCs), and their integration into the neuronal circuitry network in the adult brain, e.g., Notch, Sonic hedgehog (Shh), mitogen-activated protein kinase (MAPK) and Eph:ephrin signaling pathways (5,8-10). In addition to signaling transduction pathways, metabolic factors serve as important modulators of neurogenesis (9). The alterations in these modulators, such as growth factors during glycolysis, oxidative phosphorylation, lipogenesis and protein synthesis may be associated with the development of neurodegenerative diseases (10-13). 
In our study, adult mice were exposed to MPTP. We then carried out transcriptomic analysis on the tissues of the SVZ and DG using RNA sequencing (RNA-Seq) technology, and observed significantly deregulated gene expression following the MPTP administration. Bioinformatics analysis using the Gene Ontology (GO) and the Kyoto Encyclopedia of Genes and Genomes (KEGG) databases was applied to interpret the related function of the differentially expressed genes. Future studies using single-cell sequencing are required to further investigate the molecular targets for neuroplasticity in the SVZ and SGZ.

\section{Materials and methods}

MPTP administration and behavioral tests. All animal experiments were performed in accordance with the guidelines on the Care of Laboratory Animals, and have been approved by the Ethics Committee of Peking Union Medical College Hospital, Beijing, China. A total of 52 adult C57BL/6J female mice (10 weeks old) weighing approximately $25 \mathrm{~g}$ were randomly assigned to 2 groups: i) MPTP-exposed group $(n=29)$ and ii) saline-treated group $(n=23)$. As previously described, MPTP hydrochloride $(20 \mathrm{mg} / \mathrm{kg}$; Sigma-Aldrich, St. Louis, MO, USA) in saline was injected intraperitoneally and handled in accordance with the published guidelines $(14,15)$. The control mice received intraperitoneal injection of an equal volume of saline. The rotarod test was performed on day 21 after the MPTP injection. All mice were pre-trained approximately 1 week before the tests. One day prior to MPTP administration, the baseline was obtained, and the test series then began at a speed of $26 \mathrm{rpm}$. Each animal received 2 trials with a rest interval of 5-10 $\mathrm{min}$, and the latencies to fall off the rod within $180 \mathrm{sec}$ were recorded and averaged. For the pole test, mice were placed head upwards on the top of one upright pole approximate $30 \mathrm{~cm}$ long and $8 \mathrm{~mm}$ in diameter. Once laid upon the top, mice oriented themselves downwards and descended to the ground. Animals also received training that consisted of 3 consecutive trials of each session. After establishing the baseline, each animal experienced 2 trials and the time to descend was recorded.

Immunostaining and immunoelectron microscopy. As previously described $(14,16), 21$ days following exposure to MPTP, under deep anesthesia, the animals were sacrificed. Immunoperoxidase staining for tyrosine hydroxylase $(\mathrm{TH})$ $(n=12)$ and immunoelectron for ultrastructure $(n=10)$ were performed. Briefly, the mice were perfused intracardially with cold $0.1 \mathrm{M}$ phosphate-buffered saline (PBS) followed by $4 \%$ paraformaldehyde (PFA). The brains were carefully removed and post-fixed at $4{ }^{\circ} \mathrm{C}$ overnight, cryoprotected in $30 \%$ sucrose for 3 days. A complete set of coronal sections containing the substantia nigra (SN) were then created at a thickness of $30 \mu \mathrm{m}$. The brain slices were then pre-treated for peroxidase activity using 3\% hydrogen peroxide $\left(\mathrm{H}_{2} \mathrm{O}_{2}\right)$ for $20 \mathrm{~min}$, and blocked with 5\% normal horse serum (NHS) (Vector Laboratories, Burlingame, CA, USA) for $1 \mathrm{~h}$. Thereafter, the selected slices were incubated with primary antibodies to TH (1:800; \#AB152; Millipore, Billerica, MA, USA) at $4^{\circ} \mathrm{C}$ overnight, and treated with biotinylated horse anti-rabbit $\operatorname{IgG}$ secondary antibody (1:200; \#PK-6200) for $1 \mathrm{~h}$, followed by biotinylated protein A and avidin-biotinylated horseradish peroxidase complex (ABC kit; \#PK-6200; all from Vector Laboratories) for a further $1 \mathrm{~h}$ at room temperature. Immunoreactive cells were visualized with 3,3'-diaminobenzidine (DAB). Finally, the sections were dehydrated through a graded series of ethanol, cleared in xylene, coverslipped, and captured on a Nikon Eclipse 90i microscope (Nikon, Tokyo, Japan). Analysis was performed using the Stereo Investigator (MBF Bioscience Inc., Williston, VT, USA) to quantify TH-positive cells in the SN (14).

For direct electron microscopy, the mice were deeply anesthetized and sacrificed by intracardiac perfusion with $2 \%$ glutaraldehyde. Anatomically matched coronal sections of the SN and striatum were removed from each mouse and further fixed overnight in $2 \%$ glutaraldehyde at $4^{\circ} \mathrm{C}$ before embedding for electron microscopy. The tissues were then washed in $0.1 \mathrm{M}$ sodium cacodylate buffer ( $\mathrm{pH} 7.4)$, post-fixed with $2 \%$ osmium tetroxide for $30 \mathrm{~min}$, quickly dehydrated through graded ethanol solutions. The brain regions of interest (SN and striatum) were then cut out from tissues identically by the same individual, and covered with fresh Epon. The resulting blocks containing the regions of interest were then trimmed, and viewed on a JEOL JEM-1400Plus transmission electron microscope (JEOL, Ltd., Tokyo, Japan).

Messenger RNA ( $m R N A)$ sequencing. Total RNA was extracted from the tissues of the SVZ and DG, and RNA integrity was examined using a NanoDrop 2000/2000c (Thermo Fisher Scientific Inc., Waltham, MA, USA). The RNA library was constructed using TruSeq ${ }^{\circledR}$ RNA LT Sample Prep kit v2 (Illumina Inc., San Diego, CA, USA) according to the manufacturer's instructions. Briefly, after poly(A)-based mRNA enrichment and RNA fragmentation, first-strand complementary DNA (cDNA) was synthesized using the First Strand Master Mix and SuperScript II, followed by second-strand cDNA synthesis using the Second Strand Master Mix. Doublestranded cDNA was end repaired, and then $3^{\prime}$ ends were adenylated and the illumina adaptors were ligated. The ligation product was amplified with 15 cycles of polymerase chain reaction. After measures of yield and fragment length distribution, libraries were sequenced using TrusSeq SBS kit v3-HS, and were generated on the HiSeq 2500 sequencing system (both from Illumina Inc.). Each sequencing library generated minimally 20 million uniquely mapping reads. Images from the instrument were processed using the manufacturer's software to generate FASTQ sequence files.

Statistical analysis. The data from the behavioral tests and immunostaining are presented as the means \pm SEM and analyzed using GraphPad Prism 6.0 software (GraphPad Software, San Diego, CA, USA) for Mac OS X. The difference between 2 groups was analyzed using a two-tailed Student's t-test, while two-way ANOVA followed by Tukey's post-hoc analysis were conducted for multiple comparisons between 2 or more groups. A p-value $<0.05$ was defined as a threshold for statistical significance.

To characterize the transcriptomic profiles, RNA-Seq was performed. Raw reads were trimmed the adaptor sequences with trim Galore. Sequencing reads were mapped to the mouse genome using TopHat 2.1.0. Cufflinks was used to calculate the gene expression values represented as fragments per kilobase per million mapped reads (FPKM). The differential gene 
A

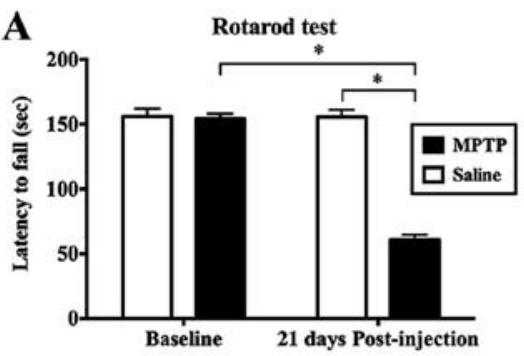

B

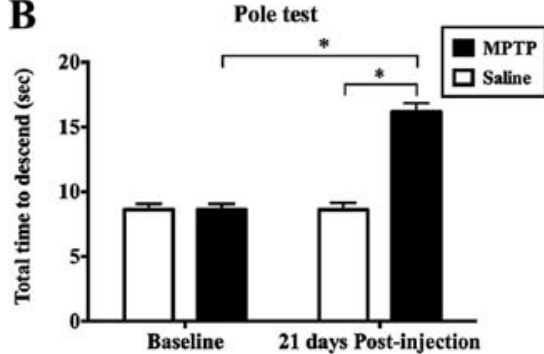

E

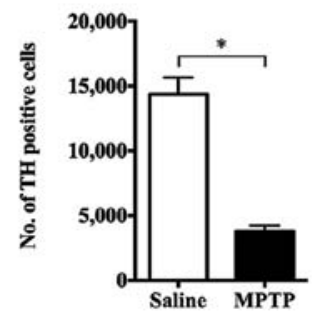

C

D

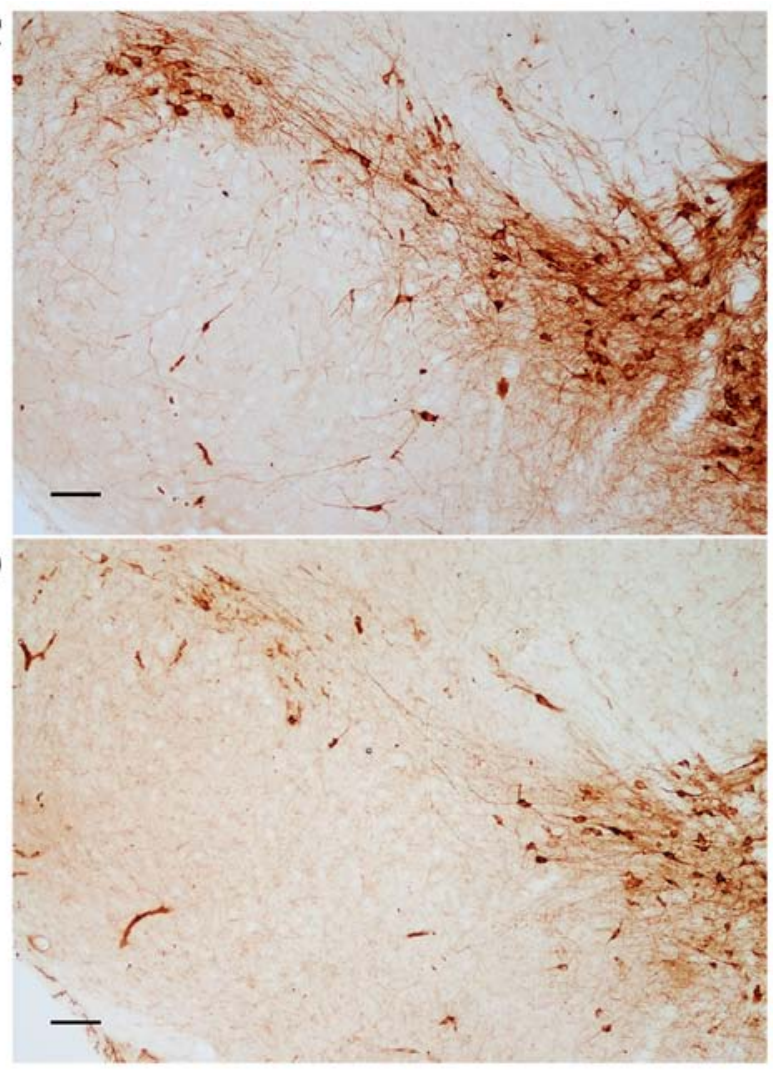

Figure 1. Behavioral tests and immunostaining of tyrosine hydroxylase (TH)-positive neurons in the substantia nigra (SN). No significant difference in motor performance in mice was observed in the (A) rotarod or (B) pole test prior to exposure to 1-methyl-4-phenyl-1,2,3,6-tetrahydropyridine (MPTP). Notably, mice administered MPTP exhibited a significant deterioration in locomotor activity 21 days after the intraperitoneal injection of the neurotoxin compared to the saline-treated controls, or the baseline. Values represent the means \pm SEM of 4-6 mice/group ( ${ }^{*} \mathrm{p}<0.05$; two-way ANOVA). When compared with the (C) salineinjected control, there was a marked reduction in TH immunoreactivity at the level of the SN in the (D) mice administered MPTP. Scale bars represent $300 \mu \mathrm{m}$. (E) Stereological analysis revealed a significantly greater reduction in the number of nigral TH-positive neurons of MPTP-exposed mice compared to the control animals 21 days after administration. Scale bars represent the means \pm SEM of 4-6 mice/group ( ${ }^{*}$ p $<0.05$; two-tailed Student's t-test).

expression analysis of RNA-Seq data was performed using Cuffdiff (17). The differential expression genes were filtered with FPKM $>1$, q-value $<0.05$ and $\log 2$ fold change $>1$ or $<-1$, and clustered using Gene Cluster 3.0. In addition, GO and KEGG analysis were conducted using the DAVID bioinformatics database. WEGO was used to plot the GO annotation results. Expression distribution and scatter plots were generated with CummeRbund package in $\mathrm{R}$ (18)

\section{Results}

MPTP-induced parkinsonism and pathological changes in mice. The rotarod and pole tests were compared between mice administered MPTP and animals receiving saline. No significant difference in behavioral performance was observed prior to MPTP. However, 21 days following exposure to MPTP, a statistically significant deterioration in locomotor activity was observed in the mice given the neurotoxin compared to the saline-treated controls. The mice with PD remained on the rotarod for significantly shorter period of time than the control animals ( $<<0.05$; Fig. 1A). For the pole test, the mice intoxicated with MPTP took a significantly longer time to complete the test ( $\mathrm{p}<0.05$; Fig. 1B).

We stereologically counted the number of dopaminergic neurons in the SN stained for TH. When compared with the saline-injected control (Fig. 1C), there was a marked reduction in $\mathrm{TH}$ immunoreactivity in the mice adminstered MPTP (Fig. 1D). The MPTP-exposed mice had an average of only $3,803.29 \pm 423.96$ dopaminergic neurons in the SN compared with the saline-infused animals which had 14,373.53 \pm 1289.03 cells expressing TH $(p<0.05$; Fig. 1E). Furthermore, we observed the ultrastructure of neurons in the SN and neurites in the striatum. Striking changes did not occur in the saline group (Fig. 2A and B). In the MPTPexposed mice, the plasma membrane of dying neurons appeared ruptured, and the perinuclear cytoplasm was severely dilated, including the endoplasmic reticulum (ER) (arrows in Fig. 2C). In addition, there were numerous enlarged dystrophic myelinated axons (asterisks in Fig. 2D) swelled, containing mitochondria with varying degrees of degeneration in the striatum of the mice administered MPTP. Notably, one greatly enlarged mitochondrion grossly deformed into a ring-shaped structure (arrow in Fig. 2E), while another one had prominently residual cristae (black arrowheads in Fig. 2F).

Differential expression of genes in the SVZ and DG of mice following exposure to MPTP. To characterize the transcriptomic profiles in mice at 4 days and 3 weeks after the MPTP administration, RNA-Seq was performed. In the SVZ, we found 106 genes significantly deregulated 
A

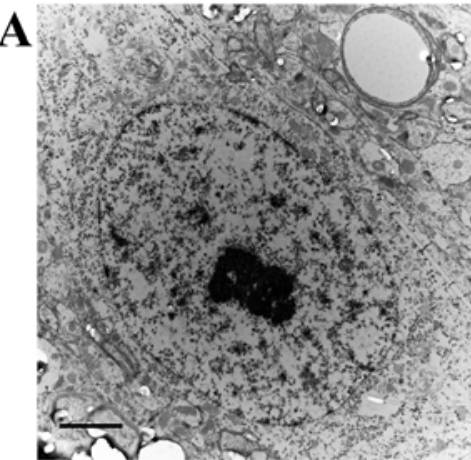

B
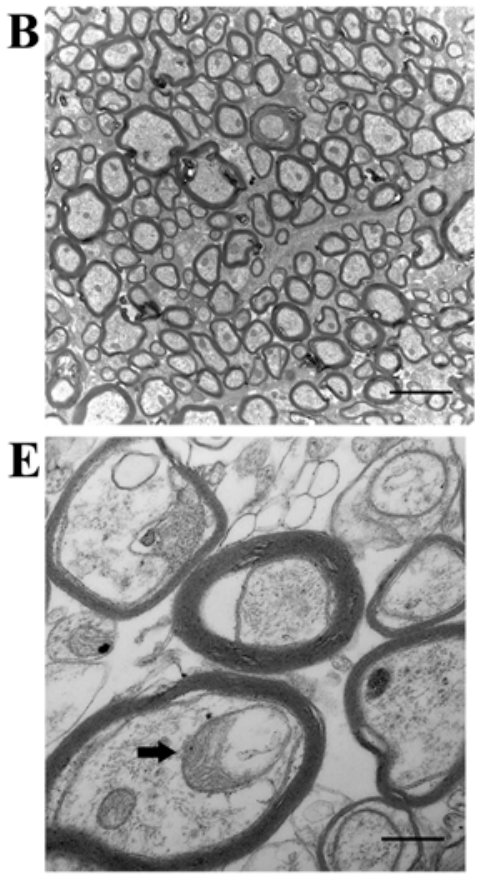
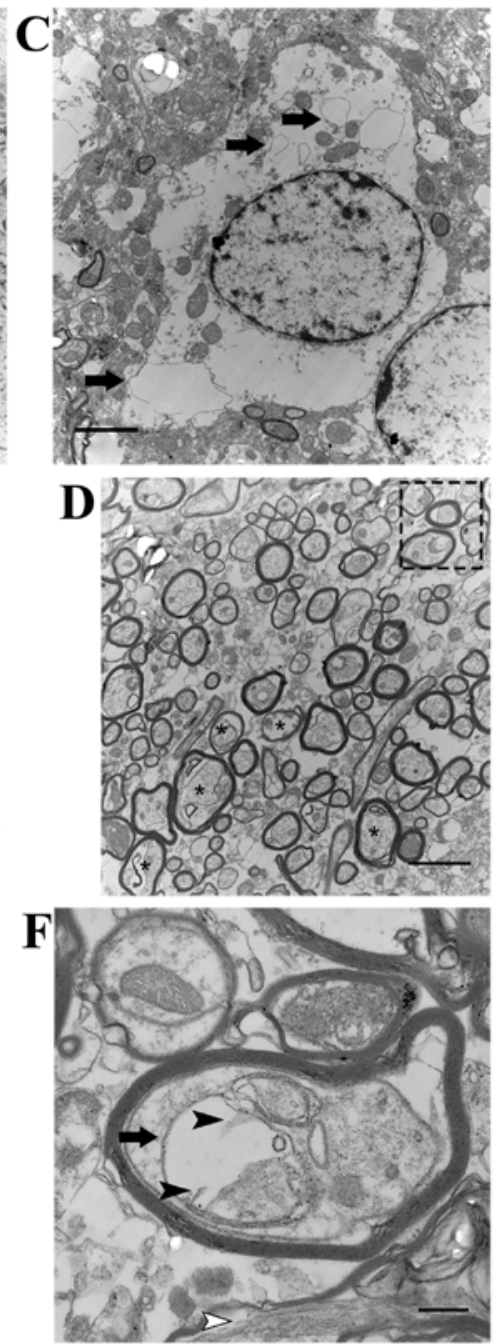

Figure 2. Ultrastructural analysis of neuronal alteration and axonal degeneration following exposure to 1-methyl-4-phenyl-1,2,3,6-tetrahydropyridine (MPTP) by transmission electron microscopy. Neuronal structures in the (A) substantia nigra (SN) and (B) axons in the striatum appeared normal in animals not administered MPTP. (C) A dying neuron in the SN from the MPTP-exposed group. Plasma membrane appeared ruptured and organelles were largely destroyed. Perinuclear cytoplasm was severely dilated including endoplasmic reticulum (ER) (arrows). (D) Axonal degeneration with increased endoneurial space was identified in the striatum of mice administered MPTP. Dystrophic myelinated axons swelled (asterisks), containing disintegrating mitochondria, but without disruption of myelin sheath. Dashed box in (D) indicates the location of the magnified view of surrounding neuropil (E). One enlarged axonal mitochondrion appeared in a ring-like shape (arrow in E) close to a normal-sized mitochondrion. Note the aberrant mitochondrion (black arrow in F) with residual cristae (black arrowheads in F). In addition, the aggregation of filamentous material was observed in the myelinated axon (white arrowhead in F). Scale bars represent $10 \mu \mathrm{m}$ in (A-D) and $2 \mu \mathrm{m}$ in (E and F) .

at different time points: 65 were upregulated and 41 were downregulated (q-value $<0.05$ compared with the normal groups; Fig. 3A). In the DG, the expression levels of a total of 98 genes were significantly modulated: 43 were upregulated, and 55 were downregulated (q-value $<0.05$ compared with the normal groups; Fig. 3B). We identified two different temporal profiles of gene expression in the SVZ (Fig. 4A): i) cluster A, genes with long-lasting upregulation [from day 4 to week 3 , e.g., pyruvate dehydrogenase kinase, isozyme 4 (Pdk4) and $\mathrm{G}$ protein-coupled receptor 17 (Gprl7)]; and ii) cluster B, genes with a marked but transient decrease and further oppositely regulated [e.g., nuclear receptor subfamily 4, group A, member 3 (Nr4a3), Fos-like antigen 2 (Fosl2), early growth response 2 (Egr2), Egr4, and solute carrier family 17 (vesicular glutamate transporter), member 7 (Slc17a7)].

Biological functions and pathway analysis of gene expression changes in the SVZ and DG. To interpret biological functions that may be altered by differential gene expression after MPTP administration, we performed GO analysis using the DAVID bioinformatics database. In the SVZ of the day 4 group, some of the biological functions were downregulated, including transcription factor activity and the transmission of nerve impulse, while the significantly enriched GO term associated with MPTP-induced changes was immune response (Fig. 4B). The increased expression of genes included in immune response indicated that this function was upregulated in the SVZ at the early stage. However, we found a significant enrichment in transcription factor activity, the transmission of nerve impulse and myelination, which were declining at day 4 but presented an opposing direction of changes 3 weeks following exposure (Fig. 5A; Table I). Additionally, other functions were observed with significant downregulation, such as cell adhesion (Fig. 5A). In the DG, a number of biological functions with considerable activation were found (Fig. 5B; Table II), including cell junction, 

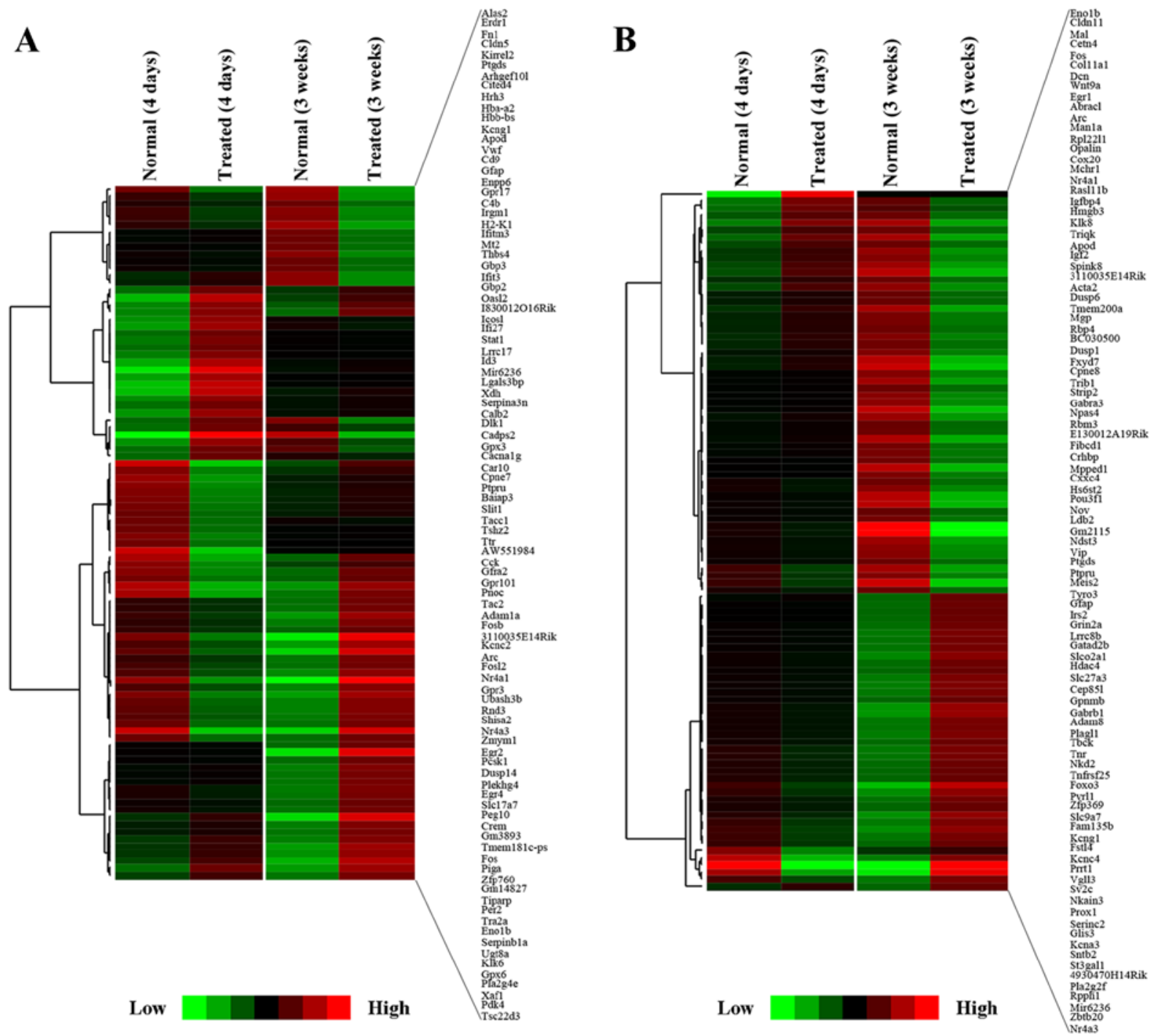

Figure 3. Gene expression signature in the subventricular zone (SVZ) and dentate gyrus (DG) 4 days and 3 weeks after 1-methyl-4-phenyl-1,2,3,6-tetrahydropyridine (MPTP) administration. Hierarchical cluster analysis performed on genes significantly altered in the (A) SVZ and (B) DG following exposure to MPTP (FPKM $>1$, fold change $>1$ or $<-1$, q-value $<0.05$ ). The cluster graphs were obtained using correlation (uncentered) similarity metric and centroid linkage clustering methods with Gene Cluster 3.0 software. The green probes correspond to downregulated genes, and the red ones corresponded to upregulated genes.

synapse and fatty acid transport. Notably, the downregulation of the functions, such as transcription activator activity, MAPK phosphatase activity, extracellular region and positive regulation of transcription, suggested a potential negative effect following exposure to MPTP (Fig. 5B).

KEGG pathway analysis was then used to reveal diverse functional entities involved in inter- as well as intra-cellular signaling cascades. Genes involved in the MAPK pathway [e.g., FBJ murine osteosarcoma viral oncogene homolog (Fos), and nuclear receptor subfamily 4, group A, member 1 ( $N r 4 a l)]$, were significantly upregulated in the SVZ 3 weeks after the MPTP administration, suggesting active signaling in this subregion (Fig. 6). In the DG, we represented significantly downregulated genes in a model of the MAPK pathway. Surprisingly, the molecular signature in response to MPTP resulted in a negative effect upon the cascade (Fig. 7). In addition to the downregulation of transcription factors (Fos and $\mathrm{Nr} 4 \mathrm{al}$ ), a marked decrease of dual-phosphatases mRNA [dual specificity phosphatase 1 (Dusp1) and Dusp6] was observed.

\section{Discussion}

In this study, using a transcriptomic approach and taking into account the time course of the effects, we identified gene expression signature in the SVZ, as well as in the DG induced by an intraperitoneal injection of MPTP. Our results demonstrated that the expression of 106 genes in the SVZ and 98 genes in the DG were modulated in the mice with PD. Immune response, transcription factor activity, transmission of nerve impulse, myelination and cell adhesion in the SVZ, and cell junction, fatty acid transport, extracellular region, and positive regulation of transcription in the DG were biological functions specifically enriched in our deregulated gene list. Overall, our data indicated tha expossure to MPTP regulated MAPK signaling cascades through changes in transcription factors, as well as MAPK phosphatases.

Genes related to neurogenesis in the SVZ. Based on the temporal effects, the expression profiles of more significantly altered 


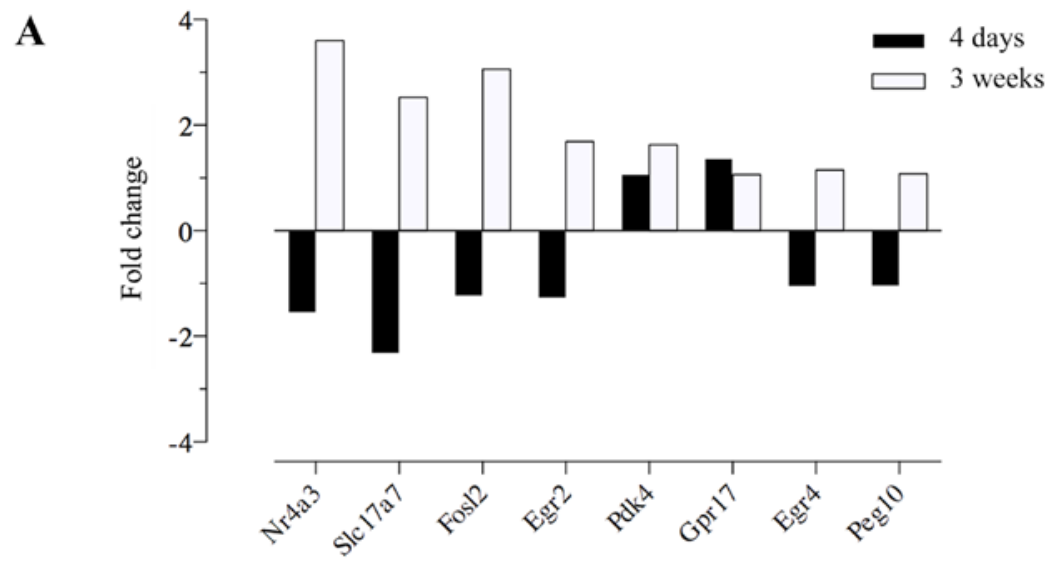

B

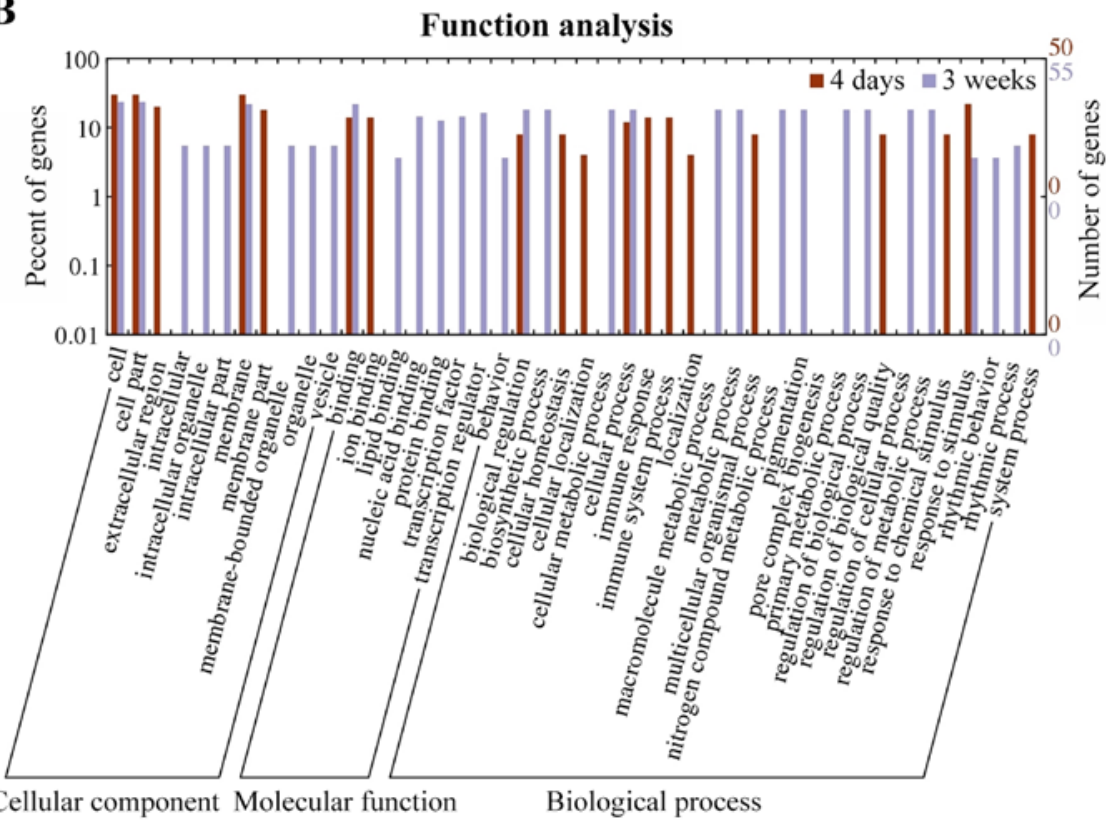

Figure 4. Gene expression patterns and Web Gene Ontology Annotation Plot (WEGO) in the 1-methyl-4-phenyl-1,2,3,6-tetrahydropyridine (MPTP)-exposed subventricular zone (SVZ) at different time points. (A) Identification of MPTP-induced changes in gene expression in the SVZ corresponding to the different temporal expression profiles. There were two different gene expression patterns: i) cluster A, genes with long-lasting upregulation throughout the experimental periods [pyruvate dehydrogenase kinase, isozyme 4 ( $P d k 4)$ and $\mathrm{G}$ protein-coupled receptor 17 ( $\mathrm{Fprl7}$ )]; followed by ii) cluster B, genes with a significant decrease at the early stage but further overrepresented 3 weeks after MPTP [nuclear receptor subfamily 4, group A, member 3 (Nr4a3), Fos-like antigen 2 (Fosl2), early growth response 2 (Egr2), Egr4 and solute carrier family 17 (vesicular glutamate transporter), member 7 (Slc17a7)]. (B) The annotation analysis of transcriptomic changes in the SVZ was plotted and compared between two datasets of day 4 and week 3 by WEGO. Gene Ontology (GO) pathways with $>3$ genes were considered significant and included in the figure. In this histogram, significantly differential genes (FPKM $>1$, fold change $>1$ or $<-1$, q-value $<0.05$ ) were compared between the day 4 and week 3 group.

genes in the SVZ were divided into two clusters. Cluster A was composed of genes with long-lasting upregualtion (e.g., $P d k 4$ and $G p r 17)$. Pdk4 encodes pyruvate dehydrogenase kinase that inactivates pyruvate dehydrogenase complex in the mitochondria (19), shunting the oxidative phophorylation of glucose to a glycolytic pathway, particularly after MPTP-induced neurodegeneration (20). Our results revealed that the MPTP-mediated increase in $P d k 4$ mRNA expression was sustained in the SVZ, indicating a metabolic switch to maintain energy demands. A recent study suggested that the selective activation of $\mathrm{Gpr} 17$ promoted neuronal differentiation and neurite elongation (21). In addition, Gprl7 can activate the intracellular phosphorylation of both extracellular signal-regulated kinase (ERK) and p38 cascades (21), which have been identified as members of the MAPK signaling pathway involved in cell proliferation, differentiation and survival $(8,9)$. Likewise, we also identified that $G \operatorname{pr} 17$ exhibits persistent upregulation, which may indicate the active signaling in the SVZ.

Cluster B consisted of genes with a marked decrease at the early stage, but further overrepresented 3 weeks after MPTP exposure. Of note, this cluster included several immediate-early genes (IEGs) (e.g., $N r 4 a 3$, Fosl2, Egr2 and Egr4) induced by multiple stressors (22-24). It is well known that DA serves as a regulatory molecule in the SVZ based on the localization of dopaminergic projections along and within this region $(25,26)$. More recent studies on dopaminergic neurons have indicated that haloperidol rapidly induces $\mathrm{Nr} 4 \mathrm{a} 3$ in the ventral tegmental area (VTA), and this is accompanied by the induction of TH and the DA transporter-mRNA (27). When genes were ranked by fold change in our study, $N r 4 a 3$ was at the top of the list 
Table I. Selected genes differentially expressed in the SVZ of mice 3 weeks after MPTP exposure.

\begin{tabular}{|c|c|c|c|c|}
\hline Accession no. & Gene symbol & Gene name & FC & q-value \\
\hline \multicolumn{5}{|c|}{ Genes related with molecular function } \\
\hline \multicolumn{5}{|c|}{ GOTERM_MF_FAT transcription factor activity, 8 genes, p-value $7.76 \mathrm{E}-3$} \\
\hline NM_010234 & Fos & FBJ osteosarcoma oncogene & 1.30 & 0.011 \\
\hline NM_001077364 & $T s c 22 d 3$ & TSC22 domain family, member 3 & 1.19 & 0.011 \\
\hline NM_010118 & Egr2 & Early growth response 2 & 1.69 & 0.011 \\
\hline NM_008037 & Fosl2 & Fos-like antigen 2 & 3.06 & 0.011 \\
\hline NM_001110850 & Crem & cAMP responsive element modulator & 1.06 & 0.040 \\
\hline NM_010444 & $N r 4 a 1$ & Nuclear receptor subfamily 4, group A, member 1 & 1.68 & 0.011 \\
\hline NM_001307989 & $N r 4 a 3$ & Nuclear receptor subfamily 4 , group A, member 3 & 3.60 & 0.011 \\
\hline NM_008036 & Fosb & FBJ osteosarcoma oncogene B & 1.14 & 0.011 \\
\hline
\end{tabular}

Genes related with biological process

GOTERM_BP_FAT transmission of nerve impulse, 5 genes, p-value 0.033

\begin{tabular}{|c|c|c|c|c|}
\hline NM_182993 & Slc17a7 & Solute carrier family 17, member 7 & 2.52 & 0.011 \\
\hline NM_007657 & $C d 9$ & CD9 antigen & 2.06 & 0.011 \\
\hline NM_010118 & Egr2 & Early growth response 2 & 1.69 & 0.011 \\
\hline NM_001205075 & Pnoc & Prepronociceptin & 1.44 & 0.019 \\
\hline NM_011674 & $U g t 8 a$ & UDP galactosyltransferase $8 \mathrm{~A}$ & 1.17 & 0.011 \\
\hline
\end{tabular}

GOTERM_BP_FAT regulation of membrane potential, 4 genes, p-value 0.026

\begin{tabular}{|c|c|c|c|c|}
\hline NM_007657 & $C d 9$ & CD9 antigen & 2.06 & 0.011 \\
\hline NM_010118 & Egr2 & Early growth response 2 & 1.69 & 0.011 \\
\hline NM_011674 & $U g t 8 a$ & UDP galactosyltransferase $8 \mathrm{~A}$ & 1.17 & 0.011 \\
\hline NM_001112813 & Cacnalg & Calcium channel, voltage-dependent, & -1.05 & 0.019 \\
\hline
\end{tabular}

GOTERM_BP_FAT axon ensheathment, 3 genes, p-value 0.019

\begin{tabular}{|c|c|c|c|c|}
\hline NM_007657 & $C d 9$ & CD9 antigen & 2.06 & 0.011 \\
\hline NM_010118 & Egr2 & Early growth response 2 & 1.69 & 0.011 \\
\hline NM_011674 & $U g t 8 a$ & UDP galactosyltransferase $8 \mathrm{~A}$ & 1.17 & 0.011 \\
\hline \multicolumn{5}{|c|}{ GOTERM_BP_FAT myelination, 3 genes, p-value 0.017} \\
\hline NM_007657 & $C d 9$ & CD9 antigen & 2.06 & 0.011 \\
\hline NM_010118 & Egr2 & Early growth response 2 & 1.69 & 0.011 \\
\hline NM_011674 & $U g t 8 a$ & UDP galactosyltransferase $8 \mathrm{~A}$ & 1.17 & 0.011 \\
\hline
\end{tabular}

Genes were grouped in 5 functional categories: transcription factor activity, transmission of nerve impulse, regulation of membrane potential, axon ensheathment and myelination. Note that some genes were included in several categories. Gene function was attributed using DAVID bioinformatics database for clustering by functional annotation, and the number of genes and p-value of each biological function were included. FC represents the fold change between gene expressions of MPTP-exposed mice vs. saline-infused animals. SVZ, subventricular zone; MPTP, 1-methyl-4-phenyl-1,2,3,6-tetrahydropyridine.

of genes, in agreement with previous studies showing that the induction of $\mathrm{Nr} 4 \mathrm{a3}$ was associated with repeated L-DOPA administration that led to motor improvement (22). The sum of the therapeutic effects indicated that IEGs, such as $\mathrm{Nr} 4 \mathrm{a} 3$ cab be regarded as a hallmark of recovery in the DA-depleted brain. Although the most enriched GO term was immune response at the early stage, in our study, the expression profiles of IEGs (Nr4a3, Egr2 and Egr4) in the SVZ demonstrated that dysfunctional dopaminergic innervation may be partially rescued. In terms of temporal dynamics, some functions referring to the genes we discussed before were also negatively impacted by MPTP at the early stage and further returned to the normal state, including transcription factor activity [Fos, Fosl2,
FBJ murine osteosarcoma viral oncogene homolog B (Fosb), $\mathrm{Nr} 4 \mathrm{al}, \mathrm{Nr} 4 \mathrm{a} 3$ and $\mathrm{Egr} 2$ ], transmission of nerve impulse (Slc17a7 and Egr2) and myelination (Egr2). Firstly, the upregulation of transcription factors (Fos, Fosl2, and Fosb) may influence the expression of various genes involved in cell proliferation and differentiation after extracellular stimuli. Particularly, Fos is currently used as a marker of neuronal activity and has been associated with a number of neural responses to acute stimuli expression. A decrease in the level of Fos was observed in age-related degeneration, as well as impaired brain development (28). Llorens-Bobadilla et al found that the active state of NSCs in the SVZ was associated with the expression of IEGs including Fos (10). Moreover, other IEGs such as $\mathrm{Nr} 4 \mathrm{al}$ 
Table II. Selected genes differentially expressed in the DG of mice 3 weeks after MPTP exposure.

\begin{tabular}{|c|c|c|c|c|}
\hline Accession no. & Gene symbol & Gene name & $\mathrm{FC}$ & q-value \\
\hline \multicolumn{5}{|c|}{ Genes related with molecular function } \\
\hline \multicolumn{5}{|c|}{ GOTERM_MF_FAT transcription activator activity, 7 genes, p-value 2.22E-3 } \\
\hline NM_007913 & Egrl & Early growth response 1 & -1.55 & 0.006 \\
\hline NM_207225 & Hdac4 & Histone deacetylase 4 & 1.16 & 0.010 \\
\hline NM_010444 & Nr4al & Nuclear receptor subfamily 4 , group A, member 1 & -1.77 & 0.006 \\
\hline NM_019740 & FoxO3 & Forkhead box $\mathrm{O} 3$ & 1.20 & 0.006 \\
\hline NM_001307989 & $N r 4 a 3$ & Nuclear receptor subfamily 4 , group A, member 3 & 1.01 & 0.010 \\
\hline NM_011141 & Pou3fl & POU domain, class 3 , transcription factor 1 & -3.65 & 0.006 \\
\hline NM_153553 & Npas4 & Neuronal PAS domain protein 4 & -2.04 & 0.006 \\
\hline
\end{tabular}

Genes related with cellular component

GOTERM_CC_FAT synapse, 7 genes, p-value 4.14E-3

$\begin{array}{lllr}\text { NM_207225 } & \text { Hdac4 } & \text { Histone deacetylase 4 } & 1.16 \\ \text { NM_001276684 } & \text { Arc } & \text { Activity regulated cytoskeletal-associated protein } & -1.67 \\ \text { NM_008067 } & \text { Gabra3 } & \text { Gamma-aminobutyric acid (GABA) A receptor, subunit alpha 3 } & -1.12 \\ \text { NM_008069 } & \text { Gabrb1 } & \text { Gamma-aminobutyric acid (GABA) A receptor, subunit beta } 1 & 0.010 \\ \text { NM_008170 } & \text { Grin2a } & \text { Glutamate receptor, ionotropic, NMDA2A (c1) } & 0.006 \\ \text { NM_009229 } & \text { Sntb2 } & \text { Syntrophin, basic 2 } & 0.006 \\ \text { NM_029210 } & \text { Sv2c } & \text { Synaptic vesicle glycoprotein 2c } & 0.006 \\ \end{array}$

GOTERM_CC_FAT extracellular region, 19 genes, p-value 6.37E-4

\begin{tabular}{|c|c|c|c|c|}
\hline NM_001313969 & Vip & Vasoactive intestinal polypeptide & -1.06 & 0.033 \\
\hline NM_001159487 & Rbp4 & Retinol binding protein 4 , plasma & -2.16 & 0.027 \\
\hline NM_008940 & $K l k 8$ & Kallikrein related-peptidase 8 & -1.33 & 0.020 \\
\hline NM_198408 & Crhbp & Corticotropin releasing hormone binding protein & -1.99 & 0.006 \\
\hline NM_177059 & Fstl4 & Follistatin-like 4 & 1.06 & 0.043 \\
\hline NM_008597 & $M g p$ & Matrix Gla protein & -1.43 & 0.022 \\
\hline NM_001122736 & $\operatorname{Ig} f 2$ & Insulin-like growth factor 2 & -2.00 & 0.006 \\
\hline NM_001190451 & $D c n$ & Decorin & -1.77 & 0.006 \\
\hline NM_183136 & Spink8 & Serine peptidase inhibitor, Kazal type 8 & -2.29 & 0.006 \\
\hline NM_009177 & St3gall & ST3 beta-galactoside $\alpha$-2,3-sialyltransferase 1 & 1.02 & 0.006 \\
\hline NM_010930 & Nov & Nephroblastoma overexpressed gene & -4.57 & 0.006 \\
\hline NM_008963 & Ptgds & Prostaglandin $\mathrm{D}_{2}$ synthase (brain) & -1.73 & 0.006 \\
\hline NM_001301353 & Apod & Apolipoprotein D & -1.31 & 0.006 \\
\hline NM_022312 & $T n r$ & Tenascin-R & 1.02 & 0.006 \\
\hline NM_030890 & Prrtl & Proline-rich transmembrane protein 1 & 1.01 & 0.006 \\
\hline NM_139298 & Wnt9a & Wingless-type MMTV integration site family, member 9A & -1.06 & 0.006 \\
\hline NM_007729 & Coll1al & Collagen, type XI, $\alpha 1$ & -1.08 & 0.020 \\
\hline NM_010517 & Igfbp4 & Insulin-like growth factor binding protein 4 & -1.22 & 0.006 \\
\hline NM_012045 & Pla2g2f & Phospholipase A2, group IIF & 1.28 & 0.006 \\
\hline \multicolumn{5}{|c|}{ GOTERM_CC_FAT cell junction, 8 genes, p-value 7.00E-3 } \\
\hline NM_001276684 & Arc & Activity regulated cytoskeletal-associated protein & -1.67 & 0.006 \\
\hline NM_021424 & Pvrll & Poliovirus receptor-related 1 & 1.18 & 0.006 \\
\hline NM_008067 & Gabra3 & Gamma-aminobutyric acid (GABA) A receptor, subunit alpha 3 & -1.12 & 0.006 \\
\hline NM_008069 & Gabrbl & Gamma-aminobutyric acid (GABA) A receptor, subunit beta 1 & 1.02 & 0.006 \\
\hline NM_008170 & Grin2a & Glutamate receptor, ionotropic, NMDA2A $(\varepsilon 1)$ & 1.02 & 0.006 \\
\hline NM_009229 & Sntb2 & Syntrophin, basic 2 & 1.18 & 0.006 \\
\hline NM_001083119 & Ptpru & Protein tyrosine phosphatase, receptor type, $U$ & -1.31 & 0.006 \\
\hline NM_029210 & $S v 2 c$ & Synaptic vesicle glycoprotein $2 \mathrm{c}$ & 1.46 & 0.006 \\
\hline
\end{tabular}

Genes related with biological process

GOTERM_BP_FAT embryonic organ development, 5 genes, p-value 0.024

NM_001159487 Rbp4 Retinol binding protein 4, plasma 
Table II. Continued.

\begin{tabular}{llrr}
\hline Accession no. & Gene symbol & \multicolumn{1}{c}{ Gene name } & FC \\
\hline NM_001307989 & Nr4a3 & Nuclear receptor subfamily 4, group A, member 3 & 1.01 \\
NM_139298 & Wrt9a & Wingless-type MMTV integration site family, & -1.06 \\
& & member 9A & 0.010 \\
NM_008937 & Prox1 & Prospero homeobox 1 & 1.01 \\
NM_007729 & Coll1al & Collagen, type XI, 1 1 & -1.08 \\
GOTERM_BP_FAT positive regulation of transcription, 11 genes, p-value 5.21E-5 & 0.006 \\
NM_009538 & Plagl1 & Pleiomorphic adenoma gene-like 1 & 0.020 \\
NM_007913 & Egr1 & Early growth response 1 & 1.28 \\
NM_010234 & Fos & FBJ osteosarcoma oncogene & 0.006 \\
NM_207225 & Hdac4 & Histone deacetylase 4 & -1.55 \\
NM_001305671 & Glis3 & GLIS family zinc finger 3 & -1.77 \\
NM_001136072 & Meis2 & Meis homeobox 2 & 1.16 \\
NM_010444 & Nr4al & Nuclear receptor subfamily 4, group A, member 1 & 0.006 \\
NM_019740 & Fox03 & Forkhead box O3 & 0.006 \\
NM_001307989 & Nr4a3 & Nuclear receptor subfamily 4, group A, member 3 & 0.010 \\
NM_011141 & Pou3f1 & POU domain, class 3, transcription factor 1 & -2.37 \\
NM_153553 & Npas4 & Neuronal PAS domain protein 4 & 0.006 \\
\hline
\end{tabular}

Genes were grouped into 6 functional categories: transcription activator activity, synapse, extracellular region, cell junction, embryonic organ development and positive regulation of transcription. Note that some genes were included in several categories. Gene function was attributed using DAVID bioinformatics database for clustering by functional annotation, and the number of genes and p-value of each biological function were included. FC represents the fold change between gene expressions of MPTP-exposed mice vs. saline-infused animals. DG, dentate gyrus; MPTP, 1-methyl-4-phenyl-1,2,3,6-tetrahydropyridine.

\section{A GO analysis of SVZ (3 weeks)}

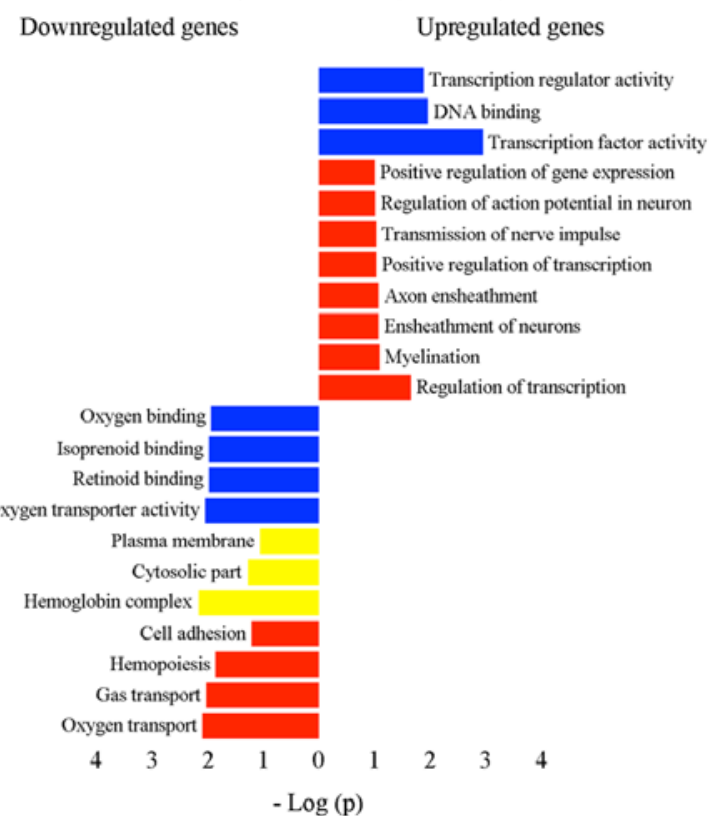

B

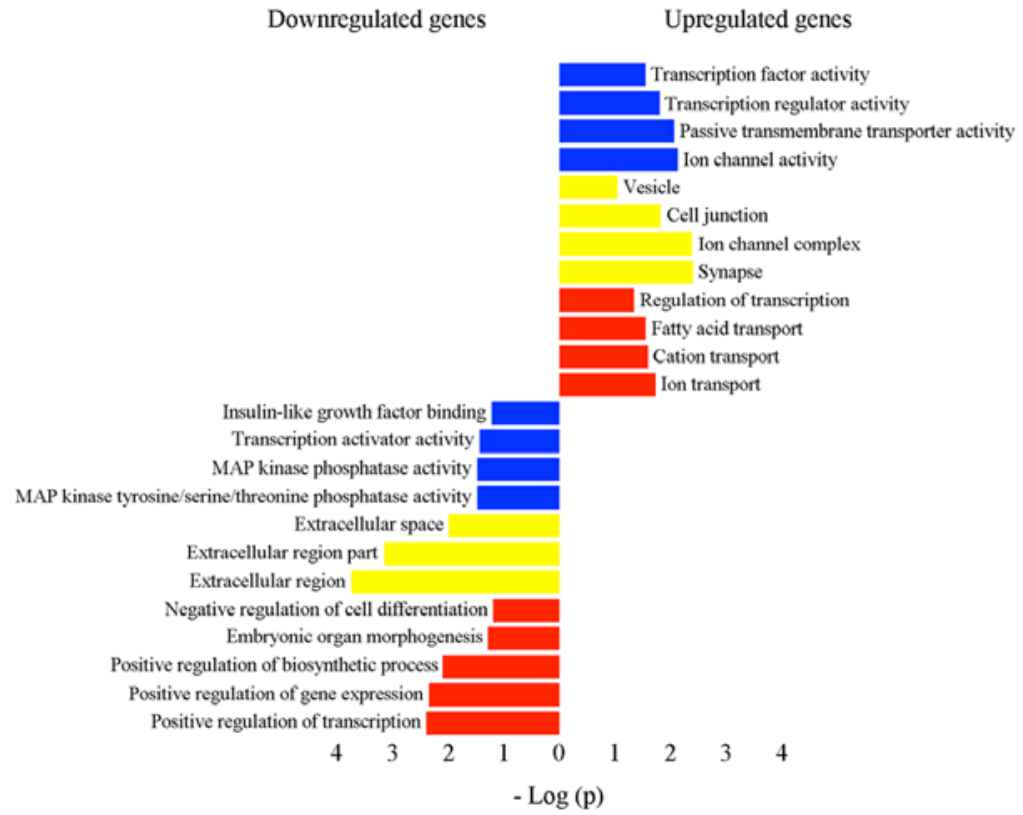

Figure 5. Gene Ontology (GO) analysis, including cellular component (yellow), molecular function (blue) and biological process (red) of transcriptomic changes in the (A) subventricular zone (SVZ) and (B) dentate gyrus (DG) 3 weeks after 1-methyl-4-phenyl-1,2,3,6-tetrahydropyridine (MPTP) administration. The analysis was performed using the functional annotation tool of the David bioinformatics database. GO pathways with $>3$ genes were considered significant if fold enrichment was $>2$-fold and the p-value $<0.05$. The vertical and horizontal axes were the GO terms and the logarithm of p-values, respectively. Improtantly, some functions observed in the SVZ after MPTP intoxication were enhanced (e.g., positive regulation of transcription, transmission of nerve impulse, and myelination), while others were inversely affected (e.g., cell adhesion). In the DG, those functions with considerable activation were identified, including cell junction, synapse and fatty acid transport. Nevertheless, transcription activator activity, mitogen-activated protein (MAP) kinase phosphatase activity, extracellular region, and positive regulation of transcription were significantly downregulated. 


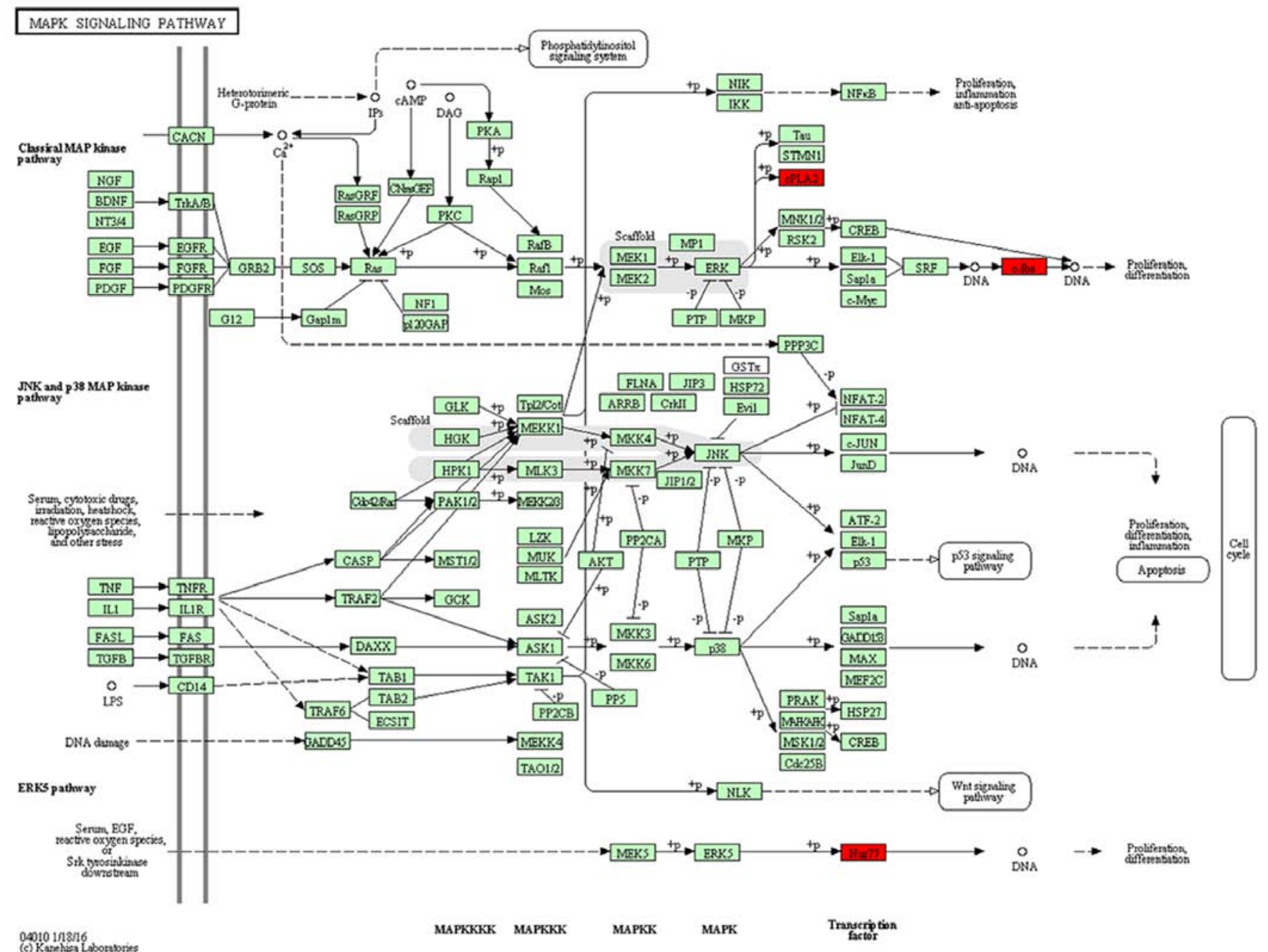

Figure 6. Synopsis of significant mitogen-activated protein kinase (MAPK) signaling pathway in the subventricular zone (SVZ) of mice exposed to 1-methyl-4-phenyl-1,2,3,6-tetrahydropyridine (MPTP) according to the Kyoto Encyclopedia of Genes and Genomes (KEGG) analysis. Specifically, related genes were upregulated (red) such as FBJ murine osteosarcoma viral oncogene homolog (Fos), and nuclear receptor subfamily 4, group A, member 1 (Nr4al).

and $\mathrm{Nr} 4 \mathrm{a3}$ encode orphan nuclear receptors that function as transcription factors (23). Nr4al has been linked to synaptic remodeling, behavioral changes, and dopaminergic loss after administrating MPTP in mice (27). The possible therapeutic role for $\mathrm{Nr} 4 \mathrm{al}$ has been manifested since neural damage was rescued by $\mathrm{Nr} 4 \mathrm{al}$ overexpression that decreased cell apoptotic rates (29) and was essential for neuronal differentiation (30). In our study, genes involved in the MAPK pathway (Fos and $\mathrm{Nr} 4 \mathrm{al}$ ) were significantly upregulated in the SVZ 3 weeks after MPTP administration. There are three major groups of MAPKs: i) ERK which is mainly activated by mitogenic stimuli, such as growth factors and hormones; ii) c-Jun N-terminal kinase (JNK) and $\mathrm{p} 38$ which are predominantly activated by stress stimuli, such as oxidative stress and inflammatory cytokines; and iii) ERK5 which is activated by both stress stimuli as well as growth factors $(9,31)$. Notably, our results displayed that since the enhanced transcription of Fos was accompanied by Nr4al, MPTP may selectively activate the ERK and ERK5 cascades in the SVZ at later stage, which may lead to the biological outcomes of cell proliferation and differentiation. Secondly, Slc17a7 and Egr2 genes may be involved in the function of glutamatergic synapse and myelination, respectively. Slc17a7 encodes the protein of vesicular glutamate transporter 1 (Vglut1) and functions in glutamate transport (32). Sconce et al suggested that the alleviation of motor deficits in mice exposed to MPTP was attributed to the recovery of extracellular Vglut1 and glutamatergic synapse (33). Others have identified a contribution of Vglut1 to glutamate release which could promote neuroblast migration and neurogenesis (34). In addition to neurotransmitter transporter, myelination that occurs primarily post-natally is essential for the efficient transmission of nerve impulses. Egr2 activates several genes that are involved in the formation and maintenance of myelin at early stage of myelination (35). Recent studies have provided evidence that oligodendrocyte progenitor cells in the SVZ migrate following exposure to stimuli and form glutamatergic synapse during the early stage of myelination process $(35,36)$. Hence, the fact that the expression of Slc17a7 and Egr2 was time-dependent has led us to speculate that neurogenesis, including synaptic formation may be initially inhibited and further improved to partially rescue the dysfunctional dopaminergic system in our parkinsonian mice. Future studies shall attempt to reveal the mechanisms underlying the temporal profiles of gene expression. 


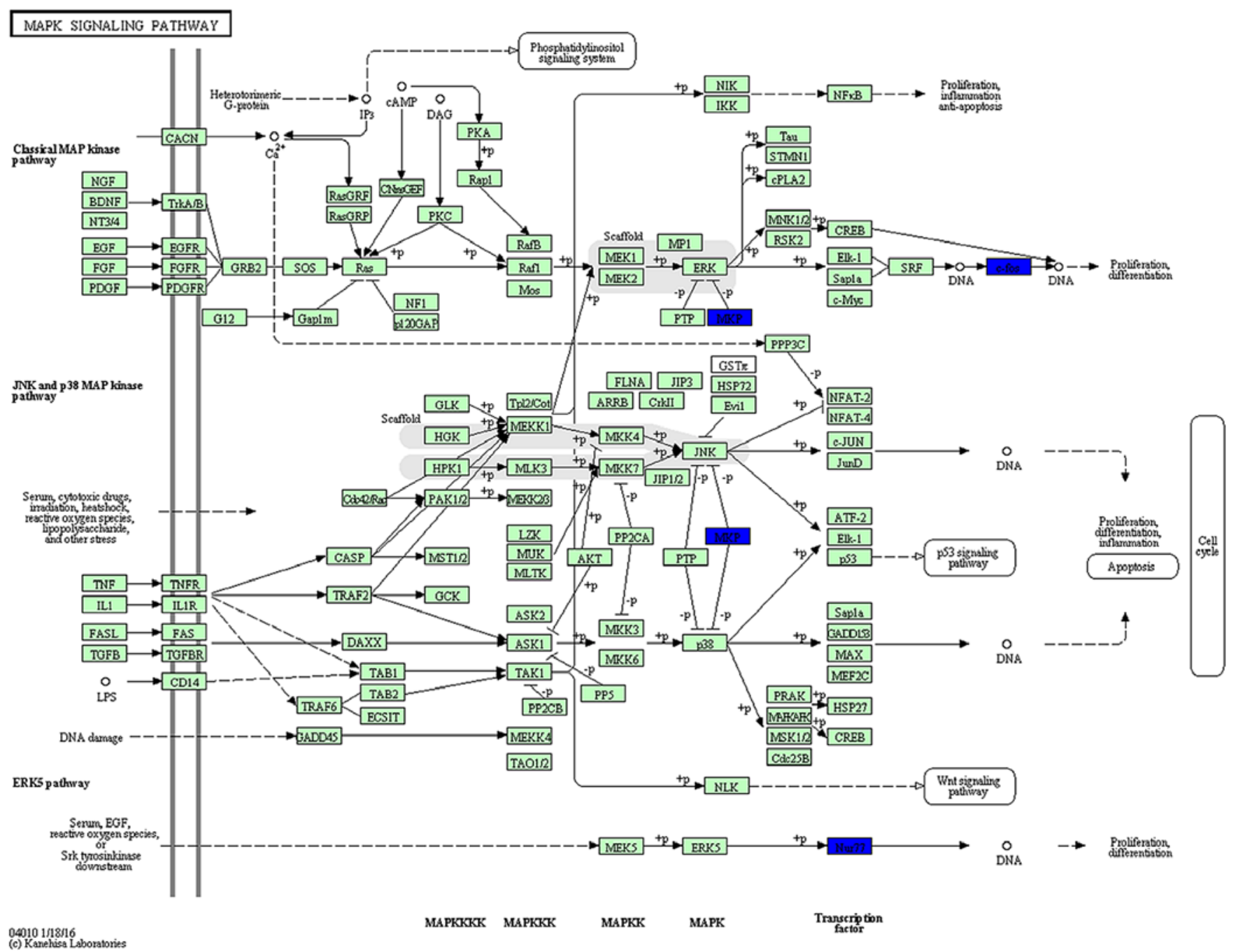

Figure 7. Synopsis of significant mitogen-activated protein kinase (MAPK) signaling pathway in the dentate gyrus (DG) of mice exposed to MPTP according to the Kyoto Encyclopedia of Genes and Genomes (KEGG) analysis. Notably, MPTP intoxication was associated with decreased expression of genes (blue) involved in transcription [FBJ murine osteosarcoma viral oncogene homolog (Fos), and nuclear receptor subfamily 4, group A, member 1 (Nr4al)] and MAPK phosphatase activity [dual specificity phosphatase 1 (Dusp1) and Dual specificity phosphatase 6 (Dusp6)].

Genes related to neurogenesis in the DG. Contrary to the expression profiles in the SVZ, the biological function of positive regulation of transcription in the DG was negatively affected by MPTP. In support of this hypothesis, the upregulation of histone deacetylase 4 (Hdac4) and the downregulation of Fos and Nr4al were observed and annotated to this function. In fact, Hdac4 encodes a protein to repress transcription, and the hight expression of Hdac4 in granule cells of the DG is associated with neurodegeneration (37). Furthermore, these changes can impact several major targets of the MAPK pathway. Specifically, the ERK and ERK5 cascades may be inactivated by MPTP at a later stage as the downstream transcription factors, Fos and Nr4al, were significantly downregulated after DA depletion. Indeed, the loss of the ERK cascade results in the depletion of neural progenitors, impairing granule cell neurogenesis in the DG $(9,38)$. Jiang et al demonstrated that the inhibition of MAPK/ERK signaling aggravated hippocampal neuronal apoptosis, decreased neurogenesis and impaired cognitive performances (39). Thus, our results support the previous findings that neurogenesis in the SGZ of the DG decreased accompanying dopaminergic denervation after MPTP epхо- sure $(2,38)$. However, we identified significant decreases in the levels of Dusp1, as well as Dusp6, which were MAPK phosphatases and negative feedback regulators of ERK cascade $(9,40)$. Consequently, by the MPTP-induced downregulation of Duspl and Dusp6, the inactivation of ERK could be reduced, leading to the compensatory reactions to the DA depletion.

In addition to the regulation of transcription, exposure to MPTP also affected genes mediating extracellular region remodeling, and cell junction, which may impact neural plasticity via cell migration or neurite outgrowth. We identified significant decreases in the expression of insulin-like growth factor 2 ( $I g f 2)$, insulin-like growth factor binding protein 4 (Igfbp 4$)$, prostaglandin $\mathrm{D}_{2}$ synthase (Ptgds) and nephroblastoma overexpressed (Nov) which could be annotated to the extracellular region. Currently, it is well accepted that Igf 2 encodes a member of the insulin-like growth factor family of proteins that can facilitate efficient signal transduction from extracellular region into the cell and promote growth and development $(11,31)$. It also acts as a physiological amplifier of glucose-mediated insulin secretion $(11,13)$, indicating a metabolic function. Moreover, the gene of Igfbp 4 encodes the 
IGF-binding proteins that have been shown to stimulate the growth promoting effects of the IGFs on cell culture $(11,12)$. On the one hand, the MPTP-mediated downregulation of Igf2 and Igfbp 4 may inhibit signal transduction, causing cell growth dysfunction. On the other hand, due to the insufficient secretion of insulin, an increase in fatty acid metabolism may begin, which was confirmed by our GO analysis. Shin et al found that quiescent NSCs in the DG maintained an active fatty acid metabolism which began to wane when these cells were activated (13). Our findings of active biological process of fatty acid transport may partially lead to the speculation that NSCs may be inactivated in the DG. Other downregulated genes related to extracellular region included collagen, type XI, $\alpha 1$ (Coll1al) (41) and decorin $(D c n)(42)$ that may have a negative impact on collagen metabolism and assembly after dopaminergic denervation. Apart from a negative effect on extracellular region remodeling, the considerable activation of cell junction and synapse were observed following exposure to MPTP, which suggested that there were some compensatory mechanisms in the DG to enable communication between neighboring cells and reduce stress. Overall, we demonstrated that epxosure to MPTP may have the potential to inhibit the expression of genes encoding proteins that could regulate extracellular matrix structure to prevent cell migration within the DG.

In conclusion, our findings demonstrated that exposure to MPTP affects gene expression in the SVZ and DG. The timedependent expression profiles in the SVZ have led us to speculate that neurogenesis may be initially inhibited and further improved in our mice with PD. Nevertheless, expression analysis has shown the dysfunction of cell proliferation and migration in the DG. Several deregulated genes that participate in the glucose and lipid metabolisms may result in metabolic switch in these two regions. It is worth emphasizing that the MAPK signaling pathway has been activated in the SVZ, while negatively regulated in the DG after MPTP administration. Future studies shall attempt to reveal the mechanisms underlying neuroplasticity in the SVZ and SGZ using single-cell sequencing to avoid cellular heterogeneity within mixed cell populations.

\section{Acknowledgements}

This study was supported by the National High Technology Research and Development Program of China (2013AA020106 and 2014AA020513), National Basic Research Program of China (2014CB541603), and National Natural Science Foundation of China (81200916).

\section{References}

1. Ming GL and Song H: Adult neurogenesis in the mammalian brain: significant answers and significant questions. Neuron 70 : 687-702, 2011.

2. Höglinger GU, Rizk P, Muriel MP, Duyckaerts C, Oertel WH, Caille I and Hirsch EC: Dopamine depletion impairs precursor cell proliferation in Parkinson disease. Nat Neurosci 7: 726-735, 2004.

3. Ohtani N, Goto T, Waeber C and Bhide PG: Dopamine modulates cell cycle in the lateral ganglionic eminence. J Neurosci 23 : 2840-2850, 2003.

4. van den Berge SA, van Strien ME, Korecka JA, Dijkstra AA, Sluijs JA, Kooijman L, Eggers R, De Filippis L, Vescovi AL, Verhaagen J, et al: The proliferative capacity of the subventricular zone is maintained in the parkinsonian brain. Brain 134: 3249-3263, 2011
5. Marxreiter F, Regensburger $M$ and Winkler J: Adult neurogenesis in Parkinson's disease. Cell Mol Life Sci 70: 459-473, 2013.

6. Aponso PM, Faull RL and Connor B: Increased progenitor cell proliferation and astrogenesis in the partial progressive 6-hydroxydopamine model of Parkinson's disease. Neuroscience 151: 1142-1153, 2008.

7. Winner B, Geyer M, Couillard-Despres S, Aigner R, Bogdahn U, Aigner L, Kuhn G and Winkler J: Striatal deafferentation increases dopaminergic neurogenesis in the adult olfactory bulb. Exp Neurol 197: 113-121, 2006.

8. Horgusluoglu E, Nudelman K, Nho K and Saykin AJ: Adult neurogenesis and neurodegenerative diseases: a systems biology perspective. Am J Med Genet B Neuropsychiatr Genet 174: 93-112, 2017.

9. Faigle R and Song H: Signaling mechanisms regulating adult neural stem cells and neurogenesis. Biochim Biophys Acta 1830: 2435-2448, 2013.

10. Llorens-Bobadilla E, Zhao S, Baser A, Saiz-Castro G, Zwadlo K and Martin-Villalba A: Single-cell transcriptomics reveals a population of dormant neural stem cells that become activated upon brain injury. Cell Stem Cell 17: 329-340, 2015.

11. Agis-Balboa RC, Arcos-Diaz D, Wittnam J, Govindarajan N, Blom K, Burkhardt S, Haladyniak U, Agbemenyah HY,Zovoilis A, Salinas-Riester G, et al: A hippocampal insulin-growth factor 2 pathway regulates the extinction of fear memories. EMBO J 30: 4071-4083, 2011.

12. Zhou R, Diehl D, Hoeflich A, Lahm H and Wolf E: IGF-binding protein-4: biochemical characteristics and functional consequences. J Endocrinol 178: 177-193, 2003.

13. Shin J, Berg DA, Zhu Y, Shin JY, Song J, Bonaguidi MA, Enikolopov G, Nauen DW, Christian KM, Ming GL, et al: Single-cell RNA-seq with waterfall reveals molecular cascades underlying adult neurogenesis. Cell Stem Cell 17: 360-372, 2015.

14. Zuo FX, Bao XJ, Sun XC, Wu J, Bai QR, Chen G, Li XY, Zhou QY, Yang YF, Shen Q, et al: Transplantation of human neural stem cells in a parkinsonian model exerts neuroprotection via regulation of the host microenvironment. Int J Mol Sci 16: 26473-26492, 2015.

15. Jackson-Lewis V and Przedborski S: Protocol for the MPTP mouse model of Parkinson's disease. Nat Protoc 2: 141-151, 2007.

16. Giasson BI, Duda JE, Quinn SM, Zhang B, Trojanowski JQ and Lee VM: Neuronal alpha-synucleinopathy with severe movement disorder in mice expressing A53T human alpha-synuclein. Neuron 34: 521-533, 2002.

17. Trapnell C, Roberts A, Goff L, Pertea G, Kim D, Kelley DR, Pimentel H, Salzberg SL, Rinn JL and Pachter L: Differential gene and transcript expression analysis of RNA-seq experiments with TopHat and Cufflinks. Nat Protoc 7: 562-578, 2012.

18. Ye J, Fang L, Zheng H, Zhang Y, Chen J, Zhang Z, Wang J, Li S, Li R, Bolund L, et al: WEGO: a web tool for plotting GO annotations. Nucleic Acids Res 34 (Web server): W293-W297, 2006.

19. Kolobova E, Tuganova A, Boulatnikov I and Popov KM: Regulation of pyruvate dehydrogenase activity through phosphorylation at multiple sites. Biochem J 358: 69-77, 2001.

20. Lee DW, Rajagopalan S, Siddiq A, Gwiazda R, Yang L, Beal MF, Ratan RR and Andersen JK: Inhibition of prolyl hydroxylase protects against 1-methyl-4-phenyl-1,2,3,6-tetrahydropyridine-induced neurotoxicity: model for the potential involvement of the hypoxia-inducible factor pathway in Parkinson disease. J Biol Chem 284: 29065-29076, 2009.

21. Daniele S, Lecca D, Trincavelli ML, Ciampi O, Abbracchio MP and Martini C: Regulation of PC12 cell survival and differentiation by the new P2Y-like receptor GPR17. Cell Signal 22: 697-706, 2010.

22. Charbonnier-Beaupel F, Malerbi M, Alcacer C, Tahiri K, Carpentier W, Wang C, During M, Xu D, Worley PF, Girault JA, et al: Gene expression analyses identify Narp contribution in the development of L-DOPA-induced dyskinesia. J Neurosci 35: 96-111, 2015.

23. Södersten E, Feyder M, Lerdrup M, Gomes AL, Kryh H, Spigolon G, Caboche J, Fisone G and Hansen K: Dopamine signaling leads to loss of Polycomb repression and aberrant gene activation in experimental parkinsonism. PLoS Genet 10: e1004574, 2014.

24. Safe S, Jin UH, Morpurgo B, Abudayyeh A, Singh M and Tjalkens RB: Nuclear receptor 4A (NR4A) family - orphans no more. J Steroid Biochem Mol Biol 157: 48-60, 2016. 
25. Björklund A and Dunnett SB: Dopamine neuron systems in the brain: an update. Trends Neurosci 30: 194-202, 2007.

26. Baker SA, Baker KA and Hagg T: Dopaminergic nigrostriatal projections regulate neural precursor proliferation in the adult mouse subventricular zone. Eur J Neurosci 20: 575-579, 2004.

27. Eells JB, Wilcots J, Sisk S and Guo-Ross SX: NR4A gene expression is dynamically regulated in the ventral tegmental area dopamine neurons and is related to expression of dopamine neurotransmission genes. J Mol Neurosci 46: 545-553, 2012.

28. Velazquez FN, Prucca CG, Etienne O, D'Astolfo DS, Silvestre DC, Boussin FD and Caputto BL: Brain development is impaired in c-fos -/- mice. Oncotarget 6: 16883-16901, 2015.

29. Xiao G, Sun T, Songming C and Cao Y: NR4A1 enhances neural survival following oxygen and glucose deprivation: an in vitro study. J Neurol Sci 330: 78-84, 2013.

30. Tomioka T, Maruoka H, Kawa H, Yamazoe R, Fujiki D, Shimoke K and Ikeuchi T: The histone deacetylase inhibitor trichostatin A induces neurite outgrowth in PC12 cells via the epigenetically regulated expression of the nur77 gene. Neurosci Res 88: 39-48, 2014.

31. Yang SH, Sharrocks AD and Whitmarsh AJ: Transcriptional regulation by the MAP kinase signaling cascades. Gene 320 : $3-21,2003$.

32. Guo Z, Zhang L, Wu Z, Chen Y, Wang F and Chen G: In vivo direct reprogramming of reactive glial cells into functional neurons after brain injury and in an Alzheimer's disease model. Cell Stem Cell 14: 188-202, 2014.

33. Sconce MD, Churchill MJ, Greene RE and Meshul CK: Intervention with exercise restores motor deficits but not nigrostriatal loss in a progressive MPTP mouse model of Parkinson's disease. Neuroscience 299: 156-174, 2015.

34. Sánchez-Mendoza E, Burguete MC, Castelló-Ruiz M, González MP, Roncero C, Salom JB, Arce C, Cañadas S, Torregrosa G, Alborch E, et al: Transient focal cerebral ischemia significantly alters not only EAATs but also VGLUTs expression in rats: relevance of changes in reactive astroglia. J Neurochem 113: $1343-1355,2010$.
35. Etxeberria A, Mangin JM, Aguirre A and Gallo V: Adult-born SVZ progenitors receive transient synapses during remyelination in corpus callosum. Nat Neurosci 13: 287-289, 2010.

36. Aguirre A, Dupree JL, Mangin JM and Gallo V: A functional role for EGFR signaling in myelination and remyelination. Nat Neurosci 10: 990-1002, 2007.

37. Whitehouse A, Doherty K, Yeh HH, Robinson AC, Rollinson S, Pickering-Brown S, Snowden J, Thompson JC, Davidson YS and Mann DM: Histone deacetylases (HDACs) in frontotemporal lobar degeneration. Neuropathol Appl Neurobiol 41: 245-257, 2015

38. Vithayathil J, Pucilowska J, Goodnough LH, Atit RP and Landreth GE: Dentate gyrus development requires ERK activity to maintain progenitor population and MAPK pathway feedback regulation. J Neurosci 35: 6836-6848, 2015.

39. Jiang P, Zhu T, Xia Z, Gao F, Gu W, Chen X, Yuan T and $\mathrm{Yu} \mathrm{H}$ : Inhibition of MAPK/ERK signaling blocks hippocampal neurogenesis and impairs cognitive performance in prenatally infected neonatal rats. Eur Arch Psychiatry Clin Neurosci 265: 497-509, 2015.

40. Banzhaf-Strathmann J, Benito E, May S, ArzbergerT, Tahirovic S, Kretzschmar H, Fischer A and Edbauer D: MicroRNA-125b induces tau hyperphosphorylation and cognitive deficits in Alzheimer's disease. EMBO J 33: 1667-1680, 2014.

41. Majava M, Hoornaert KP, Bartholdi D, Bouma MC, Bouman K, Carrera M, Devriendt K, Hurst J, Kitsos G, Niedrist D, et al: A report on 10 new patients with heterozygous mutations in the COL11A1 gene and a review of genotype-phenotype correlations in type XI collagenopathies. Am J Med Genet A 143A: 258-264, 2007.

42. Buraschi S, Neill T, Goyal A, Poluzzi C, Smythies J, Owens RT, Schaefer L, Torres A and Iozzo RV: Decorin causes autophagy in endothelial cells via Peg3. Proc Natl Acad Sci USA 110: E2582-E2591, 2013. 